\title{
Extension of a theory of predictive behavior in young children to the effects of intertrial interval duration'
}

RICHARD S. BOGARTZ

UNIVERSITY OF ILLINOIS

Twenty-five 4- and 5-year-old children predicted the next event in a 100-trial single altemation sequence. Each child received a different 50:50 random sequence of long and short intertrial interval durations. A model based on a theory emphasizing attention and short term memory processes gave a detailed quantitative description of the data. Transition relative frequencies across long and short intertrial intervals were interpreted theoretically as revealing greater guessing rates following long intervals, thus confirming the hypothesized role of a fallible short term memory subject to decay or interference effects.

Assume that a young child's prediction of the next event in a single alternation sequence passes a trace $t_{r}$ of that predictive response (PR) to a short-term memory store which holds only the most recently passed trace. The next event may elicit attending to and encoding of that event, passing an event trace $t_{e}$ to the store and removing the $t_{r}$. At any time a Distractor may pass a null trace to to the Store. Finally, occurrence of the cue to make the next PR transfers the Store's contents to a Generator which applies a transformation rule to $t_{r}$ or $t_{e}$ and a guessing rule to $t_{0}$. The information in the Generator then passes to a Predictor which produces the PR.

The Attender and Encoder are both included since behavioral evidence suggests that young children can attend to an event without encoding it. The notion of a Generator with rules permits extension of the theory to prediction of other recurrent, noncontingent, or conditional frequency event schedules.

Assume Generator rules such that transfer of the trace of one PR results in the Predictor emitting an alternate PR, transfer of an event trace results in prediction of the alternate event, and transfer of $t_{0}$ results in unbiased, random guessing. Then, if $\alpha, \beta$, and $\gamma$ are respectively the constant probabilities that a $t_{e}, t_{r}$, and $t_{0}$ are passed to the Generator, the sequence of correct responses and errors will be a two-state firstorder Markov chain with stationary transition probabilities $\mathrm{p}_{\mathrm{cc}}$ $=1-\gamma / 2, p_{c e}=\gamma / 2, p_{e c}=\alpha+\gamma / 2$, and $p_{e e}=\beta+\gamma / 2$, where $p_{i j}$ is the conditional probability of a response of type $j$ on trial $n+1$ given a response of type $i$ on trial $n$ (Bogartz, 1966).

This model applies well to prediction of a single alternation sequence by preschool children (Bogartz, 1966). Extension of the theory to varying intertrial interval durations and a test of the extension will now be given.
Table 1. Mean Performance Curve in 20-Trial Blocks

\begin{tabular}{lccccc} 
Block & 1 & 2 & 3 & 4 & 5 \\
\hline Obs. $_{\text {Pred A }}$ & .796 & .788 & .788 & .754 & .712 \\
Pred G $_{\text {G }}$ & .751 & .758 & .758 & .758 & .758 \\
\hline
\end{tabular}

If $t_{0}$ is any content of the memory other than a $t_{r}$ or $t_{e}$, it is natural from either an interference or a decay point of view to assume that the longer the intertrial interval (ITI), the greater the probability that a $t_{0}$ will enter the store. Consider the case where two ITIs, long (L) and short (S), are presented in a 50:50 random sequence. There will be two sets of transition probabilities, one for each interval. That is, we obtain the matrices

$$
\begin{array}{llllll} 
& c_{n+1} & e_{n+1} & & c_{n+1} & e_{n+1} \\
c_{n}+L & 1-\gamma_{L} / 2 & \gamma_{L} / 2 & c_{n}+S & 1-\gamma_{S} / 2 & \gamma_{S} / 2 \\
e_{n}+L & \alpha_{L}+\gamma_{L} / 2 & \beta_{L}+\gamma_{L} / 2 & e_{n}+S & \alpha_{S}+\gamma_{S} / 2 \beta_{S}+\gamma_{S} / 2 \\
\text { and } & &
\end{array}
$$

wherein, as before, $\alpha_{k}, \beta_{k}$, and $\gamma_{k}(k=L, S)$ are the probabilities of a $t_{e}, t_{r}$, and $t_{0}$ being passed to the generator following an ITI of type $k$.

These assumptions and the 50:50 random sequence of $L$ and S ITIs imply that the sequence of correct responses and errors will be a two-state Markov chaln with transition probabilities $p_{i j}=\left(p_{i j L}+p_{i j S}\right) / 2$, where $p_{i j L}$ and $p_{i j S}$ are the corresponding entries in the displayed transition matrices. Thus the data can be initially analyzed without consideration of the ITI variation, providIng a test of the Markov chain model for alternation prediction. Also, sensitivity to variation in ITI duration can be assessed using the observed transition relative frequencies corresponding to the matrices above. Finally, a new set of sequential statistics, described below, can be compared with predicted values, testing the detailed applicability of the extension to ITI variation.

Table 2. Relative Frequency of 3-Tuples

\begin{tabular}{lllllllll} 
3-Tuple & ccc & cce & cec & cee & ecc & ece & eec & eee \\
\hline Obs. & .530 & .107 & .085 & .046 & .107 & .026 & .046 & .053 \\
Pred $_{A}$ & .529 & .103 & .080 & .048 & .109 & .026 & .051 & .055 \\
Pred $_{G}$ & .523 & .109 & .076 & .056 & .111 & .023 & .057 & .042 \\
\hline
\end{tabular}




\section{Method}

The Ss were 25 four and five year old preschool children. Each was taken to an experimental room and seated at a table, opposite $\mathrm{E}$. On the table was a stack of 102 $4 \times 6$ file cards concealed behind a small black box which could hold the entire stack. Centered on each card was a $1.5 \times 2.0 \mathrm{in}$. rectangular patch of red and green tape. The colors in the stack alternated (RGRG... or GRGR. . .). The $S$ was shown the first two cards in the stack, one after the other, after being asked to name the colors on the two cards. Following correct naming of the two colors, a $6 \mathrm{~V}$ buzzer was sounded briefly and the child was told that each time he heard the buzzer he was to guess quickly the next color in the stack.

On each of 100 trials, following each buzz, $\mathrm{S}$ made his prediction, E removed the top card from the stack, turned it color side up in front of $S$ for about $1 \mathrm{sec}$, and then placed it color side down in the box. The buzzer sounded for $.3 \mathrm{sec}$ every $8 \mathrm{sec}$, except when the $\mathrm{E}$ depressed a foot switch which opened the buzzer circuit. If this occurred, the interbuzz interval was increased from $7.7 \mathrm{sec}$ to $15.7 \mathrm{sec}$. A different random sequence of long and short intervals was used with each child; thus, approximately half the intervals were long and half short. Results

The initial analyses, which do not involve the ITI variation, are presented in Tables 1,2 , and 3 . The observed statistics are averaged individual values. The Pred $_{G}$ values were obtained by using one set of parameters for

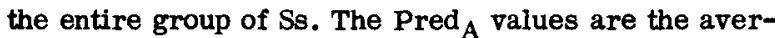
ages of the predicted values obtained with individual parameter estimation for each S. Table 1 shows the mean relative frequency of correct responses in successive blocks of 20 trials. Table 2 shows the relative frequencies with which the eight possible 3-tuples occurred in the 25 100-trial protocols. Table 3 shows the observed and predicted mean values of $r_{j}$, the number of error runs of length $j_{\text {, and }} \mathrm{C}_{\mathrm{k}}$, the number of joint occurrences of two correct responses $k$ trials apart. These results not only replicate the detailed fit of this model

Table 3. Observed and Predicted Mean Values of $r_{j}$, the Number of Error Runs of Length $j$, and $C_{k}$, the Number of Joint Occurrences of Two Correct Responses $k$ Trials Apart

\begin{tabular}{lrrrrrrr} 
& Pred $_{G}$ & Pred $_{A}$ & Obs. & & Pred $_{G}$ & Pred $_{A}$ & Cbs. \\
\hline$r_{1}$ & 7.78 & 8.28 & 8.88 & $C_{1}$ & 62.90 & 63.17 & 63.00 \\
$r_{2}$ & 3.28 & 2.67 & 2.00 & $C_{2}$ & 58.99 & 60.25 & 60.32 \\
$r_{3}$ & 1.38 & 1.07 & 1.40 & $C_{3}$ & 57.56 & 58.82 & 58.12 \\
$r_{4}$ & .58 & .50 & .68 & $C_{4}$ & 56.76 & 57.87 & 57.96 \\
$r_{5}$ & .25 & .26 & .12 & $C_{5}$ & 56.11 & 57.11 & 56.84 \\
\hline
\end{tabular}

Table 4. Observed and Predicted

Relative Frequency of the 3, 2-Tuple

\begin{tabular}{|c|c|c|c|c|c|c|c|}
\hline 3,2-Tuple & Obs. & Pred $_{A}$ & Pred $_{G}$ & 3,2-Tuple & Obs. & Pred $_{A}$ & Pred $C$ \\
\hline$c S c S c$ & .156 & .166 & .164 & $c L \subset S c$ & .137 & & \\
\hline c ScLc & .123 & .133 & .130 & $c L c L c$ & .714 & .108 & .103 \\
\hline$c S c S e$ & .016 & .012 & .013 & $c L \subset S e$ & .006 & .010 & .010 \\
\hline c SeL e & .054 & .045 & .047 & $c L c L e$ & .031 & & \\
\hline$c S e S c$ & .009 & .009 & .008 & $c \mathrm{LeSc}$ & .032 & .030 & .028 \\
\hline c SeLc & .011 & .009 & & $c L e L c$ & .033 & & .030 \\
\hline c SeSe & .004 & .005 & .006 & & .018 & & .023 \\
\hline e Le & .004 & .005 & .006 & $c L e L e$ & .021 & .020 & .021 \\
\hline e $S$ & .025 & .030 & .030 & $e \mathrm{~L} c \mathrm{Sc}$ & .030 & .0 & .032 \\
\hline$e S c L c$ & .027 & .022 & .024 & e $L c L c$ & .025 & .02 & .025 \\
\hline e $S$ & .003 & .003 & .002 & & .004 & & .003 \\
\hline e S & .011 & .010 & .009 & e $L \subset L e$ & .009 & .009 & .009 \\
\hline e $S$ e $S$ c & .011 & .011 & .014 & e $L$ e $S$ c & .013 & .013 & .013 \\
\hline & .011 & .013 & .015 & e $L$ e $L c$ & .011 & .013 & .014 \\
\hline & .016 & .015 & .012 & e $\mathrm{L}$ e $\mathrm{S}$ e & .014 & .013 & .011 \\
\hline & .014 & .013 & .011 & $e L \in L e$ & .008 & .013 & .010 \\
\hline
\end{tabular}

presented in a previous report, but yield values of the statistics surprisingly close to those found in that previous study (Bogartz, 1966).

To evaluate the ITI effects, the relative frequencies of transitions across $\mathrm{L}$ and $\mathrm{S}$ intervals were obtained. These were

$\begin{array}{lrrrrrr} & c_{n+1} & e_{n+1} & & & c_{n+1} & e_{n+1} \\ c_{n}+L & .734 & .266 & & c_{n}+S & .926 & .074 \\ e_{n}+L & .594 & .406 & \text { and } & e_{n}+S & .555 & .445\end{array}$

Recalling the theoretical matrices, we estimate the group mean probability of a $t_{0}$ being passed to the generator after a long ITI by $2 \mathrm{p}_{\mathrm{ceL}}=.532$ and the corresponding value for a short ITI by $2 \mathrm{p}_{\mathrm{ces}}=.148$. Thus, the expected effects of the ITI variation on the parameter reflecting the guessing probability were obtained, indicating that guessing increases with greater opportunity for trace decay or interference to occur.

To further evaluate the fit of the extended model, the observed and predicted mean relative frequencies of the 32 possible 3, 2-tuples were obtained (Table 4). A 3,2tuple is defined as the occurrence of a specified 3-tuple of responses on three consecutive trials in conjunction with a given pair of ITIs separating the three responses. The results in Table 4 provide additional evidence supporting the extension of the theory to variation in the ITI.

\section{References}

Bogartz, R. S. Test of a theory of predictive behavior in young children. Psychon. Sci., 1966, 4, 433-434.

\section{Note}

1. These data were collected at the University of Iowa. I am indebted to John M. Love for his assistance. 